\section{Custo da Fratura Osteoporótica de Fêmur no Sistema Suplementar de Saúde Brasileiro}

\section{RESUMO}

Objetivo: Estimar custo direto durante hospitalização para fratura osteoporótica de fêmur no sistema privado de saúde brasileiro, pela perspectiva das empresas de planos de saúde. Métodos: Estudo transversal e retrospectivo sobre custos hospitalares em pacientes acima de 50 anos com fratura osteoporótica de fêmur, entre julho 2003 e junho 2004. A amostra estudada foi extraída da base de dados de processamento eletrônico das faturas de pacientes beneficiários de planos de saúde. Resultados: Houve 129.611 pacientes com diagnóstico de osteoporose. A incidência de fratura osteoporótica de fêmur foi 4,99\% (mulheres). A média de permanência hospitalar foi 9,21 dias (2,13 dias na UTI). O custo médio total da hospitalização foi de R\$ 24.000. O maior componente de custo foi atribuído ao material médico (61\%). O impacto econômico da fratura osteoporótica de fêmur foi estimado em R\$ 12 milhões. Conclusão: Os custos do tratamento da fratura osteoporótica de fêmur são consideráveis no sistema privado de saúde brasileiro. Este estudo destaca a minimização de custos para empresas de planos de saúde caso a fratura osteoporótica de fêmur possa ser evitada. (Arq Bras Endocrinol Metab 2005;49/6:897-901)

Descritores: Osteoporose; Fratura osteoporótica; Custo econômico; Custo da doença

\begin{abstract}
Cost of Osteoporotic Hip Fracture in the Brazilian Private Health Care System.

Objective: To assess the direct cost during hospitalization for an osteoporotic hip fracture in Brazilian private health care system, by health plan companies' perspective. Methods: We conducted a crosssectional and retrospective study on costs of medical resources in patients above 50 years with an osteoporotic hip fracture, under hospital treatment, between July 2003 and June 2004. The study sample was collected from electronic claims databases of patients enrolled in Brazilian health plans. Results: There were 129,611 patients with osteoporosis diagnosis. The incidence of osteoporotic hip fracture was $4.99 \%$ (women). The mean length of hospital stay was 9.21 days (2.13 days in ICU). The total mean cost of hospitalization was R\$24,000. The largest cost component was attributable to medical device (61\%). The economic burden of osteoporotic hip fracture to health plan companies was estimated in R\$12 million. Conclusion: The costs of treating osteoporotic hip fracture are substantial in Brazilian private health care system. This study highlights the savings to health plan companies if an osteoporotic hip fracture can be avoided. (Arq Bras Endocrinol Metab 2005;49/6:897-901)
\end{abstract}

Keywords: Osteoporosis; Osteoporotic fracture; Economic cost; Cost of illness artigo original

\author{
Denizar Vianna Araújo \\ Juliana $H$. A. de Oliveiva \\ Oswaldo Luis Bracco
}

Departamento de Medicina Interna (DVA), Universidade do Estado do Rio de Janeiro (UERJ), Rio de Janeiro, RJ; e Centro Paulista de Economia da Saúde,

Universidade Federal de São

Paulo - Escola Paulista de Medicina (DVA, JHAO, OLB), São Paulo, SP.

Recebido em 14/01/05

Revisado em 07/06/05 Aceito em 15/07/05 
O STEOPOROSE É CARACTERIZADA pela redução da força óssea, predispondo a um maior risco de fratura e diagnosticada quando a densidade mineral óssea for igual ou abaixo de 2,5 desvios-padrão da densidade média local para o adulto jovem (T score) (1). Abaixo dessa densidade óssea, o risco de fratura não traumática aumenta de forma não linear. Os fatores que aumentam o risco de a mulher apresentar osteoporose e fraturas não traumáticas são: idade pós-menopausa, raça caucasiana ou asiática, baixo peso, baixo índice corporal, menopausa precoce, menarca tardia, sedentarismo, história prévia de fratura após os cinqüenta anos, história familiar de osteoporose, ingesta deficiente de cálcio, baixa exposição ao sol, doenças que afetem o metabolismo ósseo, como o hiperparatireoidismo primário e o uso crônico de corticosteróide.

A prevalência da osteoporose está aumentando em todos os países, em conseqüência do envelhecimento populacional. A estimativa de osteoporose em mulheres de origem caucasiana, na fase pós-menopausa, era de $20 \%$ na população dos Estados Unidos no início da década de 90, segundo critério diagnóstico da Organização Mundial de Saúde.

A complicação mais temida da osteoporose é a fratura proximal de fêmur. Esta fratura resulta em mortalidade $15 \%$ maior no primeiro ano pós-fratura comparativamente ao grupo não afetado de idade similar (2). Este tipo de fratura representa perda significativa da autonomia dos pacientes. Cerca de metade dos pacientes torna-se incapaz de deambular e um quarto necessita de cuidado domiciliar prolongado (3). O risco da fratura proximal de fêmur nas mulheres caucasianas, na fase pós-menopausa é de 14-17\% (4). A incidência de fraturas do fêmur aumenta exponencialmente com a idade. Os pacientes com fratura prévia são duas a cinco vezes mais susceptíveis a fraturas futuras do que indivíduos sem fraturas (5).

Além do impacto na morbidade-mortalidade, a fratura osteoporótica apresenta impacto socioeconômico relevante. Morales-Torres e cols. estimaram o impacto econômico da osteoporose na América Latina (6). Os pesquisadores realizaram revisão da literatura e pesquisa com especialistas para obter estimativas da morbi-mortalidade e utilização de recursos relacionado à osteoporose no período entre 1980 e 2003. A incidência da fratura proximal de fêmur identificada na cidade de Marília, São Paulo, foi de 343 casos por 100.000 pessoas de ambos os sexos, acima de 60 anos de idade. Os autores não identificaram estudo sobre utilização de recursos e fratura proximal de fềmur na América Latina. Os pesquisadores estimaram o custo direto de um episódio agudo com hospitalização para tratamento de fratura proximal de fêmur no Brasil como sendo US\$ 5.500, sem mencionar se o tratamento foi realizado no sistema público ou privado.

As publicações sobre o impacto econômico da fratura osteoporótica de fêmur proximal no Brasil são escassas. Silva estimou as despesas relativas às fraturas de fêmur em mulheres acima de cinqüenta anos no Sistema Único de Saúde (SUS), no ano 2001 (7). A autora utilizou a estimativa conservadora de 12.750 casos de fratura de fêmur em mulheres acima de cinqüenta anos no ano 2001 para estimar o "custo médio" de cerca de R\$ 1.700,00.

O Sistema Suplementar de Saúde representa o setor privado de assistência à saúde no Brasil, onde operadoras de planos de saúde contratam prestadores de serviços de saúde (hospitais, clínicas, consultórios, laboratórios) para atendimento de seus beneficiários. O Sistema é regulado pela Agência Nacional de Saúde Suplementar e regido pela Lei Federal 9.656/98.

Não há publicação, até o momento, sobre custo de osteoporose e seu principal desfecho, a fratura osteoporótica de fêmur proximal no Sistema Suplementar de Saúde.

O objetivo primário deste estudo foi estimar e valorar os recursos consumidos no atendimento aos pacientes acima de cinqüenta anos de idade na fase aguda da fratura osteoporótica de fêmur proximal no Sistema Suplementar de Saúde Brasileiro, sob a perspectiva das Operadoras de Planos de Saúde. O objetivo secundário foi extrapolar os dados da amostra para o universo de vidas sob cobertura no Sistema Suplementar de Saúde e estimar o impacto econômico desta condição para este Sistema.

\section{MÉTODOS}

\section{Desenho do Estudo e Características dos Pacientes}

Estudo transversal, retrospectivo sobre a estimativa de custos para o tratamento agudo da fratura osteoporótica do fêmur proximal e as complicações ocorridas durante a internação, nos pacientes de ambos os sexos acima de 50 anos atendidos no Sistema Suplementar de Saúde Brasileiro, no período de julho de 2003 a junho de 2004.

Os dados foram coletados pela empresa True Measure Research do grupo Connect Med, responsável pela transmissão eletrônica de contas médicas entre operadoras de planos de saúde e prestadores de serviços médicos (hospitais, clínicas e laboratórios de exames complementares). 
A True Measure Research realiza transmissão eletrônica de contas médicas entre 24 operadoras de planos de saúde (Seguradoras, Medicinas de Grupo, Auto-Gestões e Cooperativas médica) e 1.867 hospitais privados concentrados no estado de São Paulo $(34,8 \%)$, Minas Gerais $(12,6 \%)$ e Rio de Janeiro (11,2\%), com volume médio de 2.344 .992 contas médicas (mês de abril de 2004).

A base de dados pesquisada para o estudo representou o universo de 3.472 .553 vidas atendidas no Sistema Suplementar de Saúde, no período de julho de 2003 a junho de 2004. Os pacientes foram identificados com diagnóstico de osteoporose pela Classificação Internacional de Doenças (CID), versão 10. Todos os prontuários foram revisados para confirmação da existência de uma fratura aguda do colo do fềmur que tenha motivado uma internação hospitalar. A base de dados analisada não contemplava o registro sobre realização de densitometria óssea pré-fratura ou dosagem de cálcio em regime ambulatorial.

Foram excluídos os pacientes que possuíam outras co-morbidades de risco para fraturas patológicas, como insuficiência renal crônica ou uso prolongado de corticosteróide, que apresentassem fraturas múltiplas ou fraturas com história de traumatismo.

\section{Utilização de Recursos}

Foram computados os recursos intra-hospitalares necessários para o tratamento agudo da fratura osteoporótica do fêmur proximal e as complicações ocorridas durante a internação. Os itens mensurados foram: atendimento na sala de urgência, diária hospitalar, sala de cirurgia, unidade de tratamento intensivo, honorário profissional (médicos, enfermeiras, fisioterapeutas), medicamento, material (prótese e órtese), taxa de comercialização da prótese e órtese, exame complementar.

\section{Valoração dos Recursos de Saúde}

Os valores monetários atribuídos aos recursos foram os preços unitários de cada item e o volume total de utilização, praticados no período entre julho de 2003 e junho de 2004, no Sistema Suplementar de Saúde.

\section{Análise Estatística}

Foi realizado ajuste de erro para permitir extrapolação dos dados da amostra para o universo de vidas sob cobertura no Sistema Suplementar de Saúde.

Erro $=\left[\left(1,96^{2} \times \mathrm{p}(1-\mathrm{p}) / \mathrm{Am}\right) \times(1-\mathrm{Am} / \mathrm{Un})\right]^{0,5}$

Onde: $\mathrm{p}=0,5$ (máximo)

Am= Amostra True Measure Research

$\mathrm{Un}=$ Universo de vidas no Brasil

\section{RESULTADOS}

A amostra de pacientes acima de cinqüenta anos, de ambos os sexos, totalizou 798.687. Deste total, 129.611 (16,23\%) apresentaram diagnóstico de osteoporose para atendimento clínico ou cirúrgico no período entre julho de 2003 e junho de 2004 . O quadro 1 apresenta os percentuais de diagnóstico de osteoporose pela CID - 10 .

Foram analisadas as ocorrências de fratura osteoporótica do fêmur proximal nas pacientes do sexo feminino (evento agudo), distribuídas em faixas etárias, a partir dos cinqüenta anos (quadro 2).

Foram analisadas as ocorrências de fratura osteoporótica do fêmur proximal nos pacientes do sexo masculino (evento agudo), distribuídos em faixas etárias, a partir dos cinqüenta anos (quadro 3).

Os pacientes com diagnóstico de osteoporose (129.611) tiveram incidência de hospitalização e atendimento em unidades de urgência, em decorrência de fraturas osteoporóticas do fêmur proximal, de 0,36 atendimento por paciente por ano. Os pacientes com osteoporose apresentaram média ambulatorial de 8,17 consultas para diagnóstico e tratamento desta condição, no período analisado.

Quadro 1. Diagnóstico de osteoporose em 129.611 pacientes acima de 50 anos.

\begin{tabular}{lcc}
\hline CID & Descrição & Prevalência \\
\hline M80 & Osteoporose com fratura patológica & $43 \%$ \\
M800 & Osteoporose pós-menopausa com fratura patológica & $17 \%$ \\
M801 & Osteoporose pós-ooforectomia com fratura patológica & $9 \%$ \\
M805 & Osteoporose idiopática com fratura patológica & $2 \%$ \\
M810 & Osteoporose pós-menopáusica & $16 \%$ \\
M811 & Osteoporose pós-ooforectomia & $2 \%$ \\
M815 & Osteoporose idiopática & $3 \%$ \\
M816 & Osteoporose localizada (Lequesne) & $3 \%$ \\
\hline
\end{tabular}

Quadro 2. Fratura osteoporótica aguda do fêmur proximal nas pacientes do sexo feminino acima de cinqüenta anos.

\begin{tabular}{lcc}
\hline Faixa etária (anos) & Ocorrências na faixa etária & Percentual na faixa etária \\
\hline $50-59$ & 3.178 em 281.249 & $1,13 \%$ \\
60-69 & 4.823 em 70.312 & $6,86 \%$ \\
Acima de 70 & 7.552 em 52.734 & $14,32 \%$ \\
Total acima de 50 & 20.191 em 404.295 & $4,99 \%$ \\
\hline
\end{tabular}

Quadro 3. Fratura osteoporótica aguda do fêmur proximal em pacientes do sexo masculino acima de cinqüenta anos.

\begin{tabular}{lcc}
\hline Faixa etária (anos) & Ocorrências na faixa etária & Percentual na faixa etária \\
\hline $50-59$ & 960 em 274.359 & $0,35 \%$ \\
60-69 & 1.502 em 68.590 & $2,19 \%$ \\
Acima de 70 & 2.176 em 51.442 & $4,23 \%$ \\
Total acima de 50 & 4.638 em 394.392 & $1,18 \%$ \\
\hline
\end{tabular}


Para o tratamento cirúrgico da fratura osteoporótica do fêmur proximal, o tempo médio de internação foi de 9,21 dias e o tempo médio em unidade de tratamento intensivo (UTI) foi de 2,13 dias.

A re-internação cirúrgica em conseqüência de outro episódio de fratura osteoporótica do fềmur proximal no mesmo período de análise foi de $12 \%$ nas pacientes do sexo feminino.

A análise dos valores relativos à internação para tratamento cirúrgico da fratura osteoporótica do fêmur proximal estão apresentados no quadro 4.

A análise comparativa da base de dados estudada com o universo de vidas sob cobertura no Sistema Suplementar de Saúde revelou que a amostra era representativa. A estatística descritiva sobre a distribuição geográfica nos diferentes estados da federação apresentou-se estatisticamente igual para o teste de hipótese $(\mathrm{p}=0,90)$. A estatística descritiva sobre a distribuição por faixa etária apresentou-se estatisticamente igual para o teste de hipótese $(\mathrm{p}=0,82)$. Finalmente, estatística descritiva sobre a distribuição por sexo apresentou-se estatisticamente igual para o teste de hipótese $(\mathrm{p}=0,96)$.

O presente estudo detectou o diagnóstico de osteoporose em 16,23\% da população acima de 50 anos, sob cobertura no Sistema Suplementar de Saúde, no período entre julho de 2003 e junho de 2004 . O desfecho da fratura osteoporótica do fêmur proximal ocorreu em $3,1 \%$ dos pacientes de ambos sexos, no mesmo período.

O cadastro de beneficiários da Agência Nacional de Saúde Suplementar registrou 38,7 milhões de beneficiários em fevereiro de 2004. O segmento acima de 50 anos representou $25 \%$ do total (cerca de 9,6 milhões de beneficiários). A partir destes dados, a estimativa do número de pacientes com diagnóstico de osteoporose no Sistema Suplementar de Saúde, acima de 50 anos de idade, era de 1,6 milhão de pessoas. A estimativa para fratura osteoporótica do fêmur proximal foi de 49,6 mil procedimentos cirúrgicos para este desfecho.

Sendo assim, a estimativa do custo direto com hospitalização para tratamento da fratura osteoporó-

Quadro 4. Valores remunerados à rede hospitalar para tratamento cirúrgico da fratura osteoporótica do fêmur proximal.

\begin{tabular}{lccc}
\hline Categoria & Valor médio (R\$) & Percentual da despesa & Item de maior impacto \\
\hline Procedimentos & $3.676,45$ & $15,29 \%$ & Cirurgia e honorário \\
Taxas e diárias & $3.745,89$ & $15,57 \%$ & Diária de UTI \\
Materiais & $14.749,50$ & $61,33 \%$ & Prótese \\
Medicamentos & $1.879,33$ & $7,81 \%$ & Antibiótico \\
Total & $24.051,17$ & $100 \%$ & \\
\hline
\end{tabular}

tica do fêmur proximal para o Sistema Suplementar de Saúde totalizou R\$ 12 milhões, no período entre julho de 2003 e junho de 2004.

\section{DISCUSSÃO}

Existe a percepção por parte dos formuladores de políticas de saúde, médicos e gestores, de que o impacto econômico com as fraturas osteoporóticas de fêmur proximal é significativo e crescente para o Sistema Suplementar de Saúde no Brasil. Apesar desta percepção, inexiste estudo sobre o real impacto deste desfecho para o Sistema Suplementar. Esta falta de informação compromete a tomada de decisão na alocação racional de recursos de saúde nas estratégias diagnósticas, preventivas e terapêuticas para a osteoporose no Brasil.

O estudo apresentado contemplou o universo do Sistema Suplementar de Saúde. Este Sistema possui características sócio-econômicas e de acesso dos seus usuários, que comprometem a extrapolação a partir dos dados do SUS. O estudo utilizou amostra representativa da população de usuários do Sistema Suplementar de Saúde. A faixa etária estudada acima de 50 anos é semelhante aos dados publicados em estudos de custo da osteoporose na literatura $(8,9)$.

Ocorreu $12 \%$ de re-internação cirúrgica nas pacientes do sexo feminino, em conseqüência de outro episódio de fratura osteoporótica do fêmur proximal no mesmo período de análise. Andrade e cols. documentaram que, apesar da evidência de alto risco de fraturas subseqüentes ao primeiro episódio, o tratamento da osteoporose não é intensificado nestas pacientes, com somente $24 \%$ recebendo terapêutica específica (10).

Haentjens e cols. estimaram em US\$ 9.534 o custo médio da hospitalização para tratamento da fratura proximal de fêmur durante coorte prospectiva conduzida na Bélgica (11). Este valor encontra-se próximo ao valor estimado no nosso estudo de R \$ 24 mil (US\$ 6.900: câmbio US\$ $1=\mathrm{R} \$ 2,90)$. Haentjens e cols. contabilizaram todos os custos diretos, além da internação, decorrentes da fratura de fềmur no período de um ano, e concluíram que os pacientes com fratura osteoporótica de fêmur apresentaram custo três vezes maior que o grupo controle. Estes resultados indicam que o maior impacto econômico pode ocorrer nos meses subseqüentes à hospitalização inicial, demonstrando a necessidade de estudos prospectivos para contabilizar estes custos, e não apenas os custos decorrentes da internação aguda pós-fratura, apresentado neste estudo. 
O nosso não é um estudo de custo da doença stricto sensu, pois estimou a fratura osteoporótica de fêmur proximal a partir de despesas incorridas no Sistema Suplementar de Saúde ao invés da estimativa, in loco (hospitais), de recursos utilizados e valoração dos mesmos, isto é, o custo real. Apesar desta limitação metodológica, os valores estimados representam a despesa atribuída às fraturas osteoporóticas de fêmur proximal para a Operadora de Plano de Saúde, que representa o "Pagador" do Sistema Suplementar de Saúde. Esta estimativa indica o impacto total na sinistralidade (parcela do orçamento das empresas comprometida com o pagamento das despesas de saúde de seus beneficiários) e orienta medidas de prevenção secundária e tratamento para minimização destes custos.

Pacientes com fraturas osteoporóticas incorrem em duas vezes mais despesas no ano seguinte ao episódio da fratura comparados com pacientes com diagnóstico de osteoporose sem fratura, e quase três vezes mais com pacientes sem osteoporose (12).

O nosso estudo pretende contribuir para a compreensão sobre o impacto da osteoporose e seu principal desfecho no orçamento das Operadoras de Planos de Saúde. Esta informação será útil na tomada de decisão sobre quais intervenções diagnósticas, preventivas e terapêuticas são custo-efetivas para minimizar este impacto no Sistema Suplementar de Saúde.

\section{AGRADECIMENTOS}

Agradecemos o auxílio financeiro do Laboratório Eli Lilly do Brasil para a elaboração deste estudo.

\section{REFERÊNCIAS}

1. (WHO) World Health Organization. Assessment of Fracture Risk and its Application to Screening for Postmenopausal Osteoporosis. WHO Technical Report Series $\mathbf{8 4 3}$. Geneva, 1994.
2. Parker MJ, Palmer CR. Prediction of rehabilitation after hip fracture. Age Ageing 1995;24(2):96-8.

3. Riggs BL, Melton III LJ. The worldwide problem of osteoporosis: Insights afforded by epidemiology. Bone 1995; 17 (suppl. 5):505-11.

4. Eddy DM, Johnston CC Jr, Cummings SR. Osteoporosis: Review of the evidence for prevention, diagnosis, and treatment and cost-effectiveness analysis. Osteoporos Int 1998;8(suppl. 4): 1-88.

5. Solomon DH, Finkelstein JS, Katz JN, Mogun H, Avorn J. Underuse of Osteoporosis Medications in Elderly Patients with Fractures. Am J Med 2003; 1 15:398-400.

6. Morales-Torres J, Gutiérrez-Ureña S. The burden of osteoporosis in Latin America. Osteoporos Int 2004; 15:625-32.

7. Silva LK. Avaliação tecnológica em saúde: densitometria óssea e terapêuticas alternativas na osteoporose pós-menopausa. Cad Saúde Pública 2003;19(4): 987-1003.

8. Gabriel SE, Tosteson ANA, Leibson CL, Crowson CS, Pond GR, Hammond CS, et al. Direct Medical Costs Attributable to Osteoporotic Fractures. Osteoporos Int 2002; 13:323-30.

9. Autier P, Haentjens P, Bentin J, Baillon JM, Grivegnée AR, Closon MC, et al. Cost induced by hip fractures: A prospective controlled study in Belgium. Osteoporos Int 2000; 11:373-80.

10. Andrade SE, Majumbar SR, Chan KA, Buist DSM, Go AS, Goodman M, et al. Low Frequency of Treatment of Osteoporosis Among Postmenopausal Women Following a Fracture. Arch Intern Med 2003; 163:2052-7.

11. Haentjens $\mathrm{P}$, Autier $\mathrm{P}$, Barette $\mathrm{M}$, Boonen S. The Economic Cost of Hip Fractures Among Elderly Women. J Bone Joint Surg Am 2001;83(4):493-500.

12. Ray NF, Chan JK, Thamer M, Melton LJ. Medical expenditures for the treatment of osteoporotic fractures in the United Sates in 1995: Report form the National Osteoporosis Foundation. J Bone Miner Res 1997; 12:24-35.

\section{Endereço para correspondência:}

Denizar Vianna Araújo

Av. Visconde de Albuquerque 1400 / 501

22450-000 Rio de Janeiro, RJ

E-mail: denizarvianna@cpes.org.br 